\title{
LA CESURA: METRO Y SENTIDO EN EL VERSO GRIEGO
}

Because of the lack of agreement about how to define the caesura in Greek verse, this paper tries to emphasize on some aspects of crucial importance for the better understanding of the concrete carrying out of Greek caesura.

On the one hand, the caesura is a main element in the metrical structure of verse; on the other, it is in close relation to its meaning and its syntax. After exposing and commenting on examples proving this relation between metre and meaning, it will be insisted on the aspects of syntax and content as basic elements when considering the importance of the caesura in Greek Verse.

A pesar de los múltiples estudios y trabajos que hoy existen sobre la poesía griega antigua, seguimos sin saber exactamente cómo era su verdadera realización. En efecto, tenemos una visión parcial de lo que realmente fue un verso griego, ya que sólo contamos con el texto escrito. No obstante, a través de un estudio de su métrica y su prosodia podemos ir más allá de las simples palabras y acercarnos a la realidad que encierra el verso'.

Tal es el caso de la cesura, cuyo papel en el verso griego nos es aún muy difícil de definir. Quizá el principal obstáculo resida en el hecho de que intentamos analizar el problema griego con conceptos y términos actuales, o, mejor dicho, proyectamos sobre el verso griego el comportamiento y la realidad de la poesía de las lenguas modernas. Por ello, a la hora de definir y delimitar la cesura griega debemos intentar hacerlo desde el punto de vista griego, debemos buscar su valor en la realización concreta de la versificación original griega.

Ya desde la Antigüedad, y en los últimos tiempos desde las obras clásicas de A. W. de Groot ${ }^{2}$ y H. Drexler ${ }^{3}$, muchas han sido las defini-

1 En este sentido son perfectamente reproducibles las palabras de C. O. Pavese, «... Ben lunghi dal condividere l'opinione prevalente tra i metricologi, che coltivano la metrica come una scienza astratta e consigliano di rinunciare completamente alla lettura metrica del versi - io credo al contrario che lo studio della metrica serva anche, e soprattutto, a sentire come i versi greci effettivamente suonavano". Cf. "Tipologia metrica greca", en Problemi di metrica classica, Génova 1978, p. 66.

2 "Wesen und Gesetze der Caesur", Mnemosyne 2, 1935, pp. 86-154.

3 "Caesur und Diaerese», Aevum 24, 1950, pp. 332-336. 
ciones que se han dado sobre el concepto de cesura en el verso griego, si bien todas ellas apuntan, aunque con distintas matizaciones, a un mismo fenómeno que básicamente consiste en que hay determinadas posiciones en el verso en las cuales se busca o es frecuente el fin de palabra ${ }^{4}$. El debate más intenso en torno a este problema se dio en los años veinte entre los que negaron y defendieron la existencia de la cesura. Frente a E. H. Sturtevant ${ }^{5}$ que sólo admitía pausa a final de verso, surge el trabajo de de Groot ${ }^{6}$ que defendió la cesura como la frontera de correspondencia más importante entre las diferentes partes del verso, esto es, la "Hauptcaesur», o cesura principal. Junto a ella estarían otros fines de palabra menos frecuentes, lo que él llama "Nebencaesur», que marcan, a su vez, la frontera de correspondencia en el interior de un miembro.

A primera vista, tales definiciones parecen señalar a la cesura como un fenómeno puramente métrico sin aparente relación con el plano del significado. Efectivamente, para la mayoría de los metricólogos antiguos $^{7}$ la cesura está determinada solamente por el final de palabra, sin tener en cuenta el ritmo o el significado del verso ${ }^{8}$. S. E. Basset ${ }^{9}$, por su parte, considera que este concepto de cesura es una invención de los gramáticos, ya que los poetas antiguos realizaban, al recitar el verso, una pausa que pedía claramente el sentido.

Como vemos no ha habido, ni hay, unanimidad para definir la cesura del verso griego. A pesar de ello, nuestro propósito no es dar una de-

${ }^{4}$ Como ejemplo baste con citar las definiciones de tres de los más conocidos manuales de métrica griega:

"There are certain places where word-end (but not always word-end in the stricter sense) is either regular or frequent, but without the licenses permitted at a pause. Such places are called Caesurae when they occur inside a basic metrical sequence», P. Maas, Greek Metre, trad. ingl., Oxford $1966_{2}$, p. 36.

"Die Stelle, an der das Wortende erstrebt wird, nennen wir Zäsur», B. Snell, Griechische Metrik, Gotinga 1982, p. 11.

"A place in the verse where word-end occurs more than casually", M. L. West, Greek Metre, Oxford 1982, p. 192.

Un comentario de estas y otras definiciones del concepto de cesura puede verse en H. Drexler, "Concetti fundamentali di metrica", RFIC 93, 1965, pp. 5-63, y, sobre todo, en A. Guzmán Guerra, "Protágoras y la cesura», EC 88, 1984, pp. 93-97, cuyas reflexiones y puntualizaciones nos parecen importantes.

"WWord-Ends and Pauses in the Hexameter", AJPh 42, 1921, pp. 289-308.

6 Op. cit.

7 Las teorías de los gramáticos antiguos sobre la cesura pueden verse recogidas y comentadas en el artículo de S. E. Bassett, "The Theory of the Homeric Caesura according to the extant Remains of the ancient Doctrine», AJPh 40, 1919, pp. 343-372.

${ }^{8} \mathrm{El}$ primero que menciona este aspecto es Mario Victorino $(64,31-66 ; 114,17$ $115,4, \mathrm{~K})$.

" "The caesura a modern chimaera», $C W 18,1926$, pp. 76-79. 
finición nueva, sino llamar la atención sobre algunos puntos que hay que tener presentes para comprender mejor la realidad poético-métrica de la cesura griega ${ }^{10}$.

Lo primero que hay que tener en cuenta es que la cesura desempeña un doble papel en el verso griego. Por una parte, es pieza fundamental de la estructura métrica "e incluso prosódica del verso y, por otra, está en íntima relación con el sentido y la sintaxis de aquél. La conjunción de estos dos valores nos da la clave para abordar de una forma más efectiva el problema.

Para el aspecto prosódico de la cesura contamos con un estudio de H. J. Mette ${ }^{12}$, quien observa que las cesuras van acompañadas de no abrevimiento de vocales largas en hiato, no elisión de vocal breve, no alargamiento de sílaba ante el grupo de muta cum liquida y de alargamiento de vocal breve, fenómenos que indican una división prosódica de las palabras del verso. En la misma línea se sitúa un artículo reciente de A. Mojena ${ }^{13}$. Tras un estudio prosódico del fin de palabra en los Idilios de Teócrito la autora concluye que en el hexámetro hay una única cesura importante desde el punto de vista estructural, localizada en el tercer pie. En esta posición son frecuentes los fenómenos fonéticos señalados por Mette, mientras que se evitan en otros lugares tradicionalmente considerados como cesuras, tales como la diéresis bucólica, la heptemímeres o la tritemímeres ${ }^{14}$.

A pesar de que somos conscientes de que no hay que pasar por alto estos datos, sin embargo no estamos totalmente de acuerdo con la definición restrictiva del estructuralismo, que considera a la cesura como un simple hecho de métrica verbal o tipológica ${ }^{15}$. La cesura como elemento estructural del verso, tanto métrico como prosódico, nos da una pauta objetiva de análisis, mientras que su interrelación con el sentido da pie

${ }^{10}$ Nuestras reflexiones, así como los ejemplos que las ilustran, están centradas en el hexámetro dactílico, si bien su aplicación es válida para otro tipo de versos.

"No olvidemos que las cesuras han desempeñado un papel fundamental en el origen del hexámetro como puntos de sutura de los kola que han dado lugar al hexámetro, cf. J. F. Vigorita, "The Indo-European origins of the Greek Hexameter and Distich», ZVS 91, 1977, pp. 288-299; B. Gentili y P. Giannini, "Preistoria e formazione dell'esametro", QUCC 26, 1977, pp. 7-51, y N. Berg, "Parergon metricum: der Ursprung des griechischen Hexameters", MSS 37, 1978, pp. 11-36, entre otros.

12 «Die Struktur des ältestem daktylischen Hexameters», Glotta 35, 1956, pp. $1-17$.

${ }_{13}$ "El problema de la cesura en la métrica griega», Minerva 5, 1991, pp. 91-98.

14 A pesar de que ésta es la cesura fundamental del hexámetro, sin embargo no se pueden ignorar otros cortes, que, aunque están menos apoyados por la prosodia, si son importantes para el sentido del verso, según podremos comprobar en los ejemplos recogidos en este artículo.

${ }^{15} \mathrm{Cf}$. S. Mariner, "Hacia una métrica estructural», RSEL 1, 2, 1971, p. 315. 
a interpretaciones subjetivas. Quizá el estudio más completo que contiene esta doble vertiente sea el de R. Beck, Meter and sense in Homeric Verse (Urbana 1971), quien en su capítulo III expone un método para describir y analizar el sentido en relación con el metro en términos de introducción y continuación de unidades de sentido. Así, concluye que los puntos fundamentales para la introducción del sentido del verso son precisamente aquellos considerados tradicionalmente como cesuras, lo que él llama positivos, mientras que hay otros que son negativos, los zeugmata tradicionales, y otros neutros.

Como muy bien indicó Beck el verso está compuesto de una jerarquía de unidades ${ }^{16}$ : palabra, cláusula, frase, etc... cuya delimitación está en principio marcada por la cesura, si bien él mismo reconoce que la plena descripción de la estructura del verso no es enteramente objetiva. Desde el punto de vista puramente métrico el verso griego se organiza en una serie de elementos estructurales, kola, definidos por la presencia de un fin de palabra, la cesura. Los hechos demuestran que la unidad de medida fundamental del verso griego no es el metro, sino los segmentos en que queda descompuesto por medio de la cesura ${ }^{17}$. Las cesuras determinan los diferentes kola rítmicos del verso, que a su vez tienen una base sintáctica o de contenido, según veremos más adelante.

Ahora bien, la cesura es algo más que un simple fin de palabra, puesto que entonces nos encontraríamos indiscriminadamente con cesuras en todos los puntos del verso, hecho que no sucede así ${ }^{18}$. Los datos estadísticos ofrecidos por E. G. O'Neill ${ }^{19}$ demuestran cómo el fin de palabra en el hexámetro es más frecuente en unos lugares que en otros, precisamente en esos puntos del verso en que tradicionalmente se considera la existencia de una cesura. Ahora bien, sus datos no tienen en cuenta la realidad sintáctica y el sentido del verso, sino sólo su estructura métrica y ritmica. Sus cifras absolutas y porcentuales se reducirían si se tuvieran en cuenta estos puntos. Tal es el caso de un estudio reciente de H. R. Barnes ${ }^{20}$ sobre la colometría del hexámetro homérico. De acuerdo con los datos que ofrece para la Iliada el fin de palabra en lo

${ }^{16}$ Op. cit., p. 44.

17 Nótese en este punto la diferencia que tradicionalmente se establece con la diéresis, que coincide con el final de un metro, frente a la cesura que se da en su interior.

18 Ténganse en cuenta aquí esos lugares del verso donde está prohibida, o al menos restringida, la cesura, los llamados zeugmata.

19 "The localization of metrical Word-Types in the Greek Hexameter: Homer, Hesiod and the Alexandrians", YCS 8, 1942, pp. 105-178.

${ }_{20}$ "The colometric Structure of Homeric Hexameter», GRBS 27, 1986, pp. 125150 ; en especial pp. 130 y ss. 
que él llama "caesural area» oscila entre el $86,9 \%$ en el tercer kolon y el $98,9 \%$ del segundo, mientras que el porcentaje se reduce considerablemente si se considera la "sense-unit».

La cesura es un "word-end" restringido tanto por el ritmo del metro como por el sentido. Como indica E. H. Sturtevant ${ }^{21}$ el punto clave para entender el problema de la cesura es su distinción del simple hecho de fin de palabra, ya que el único punto común entre ambos es que toda pausa de sentido requiere un fin de palabra. Incluso, como ya hemos mencionado, la cesura está determinada y en estrecha relación con la estructura prosódica del verso.

En el fondo está el problema de la definición de la unidad métrica básica, del kolon. Para el caso concreto del hexámetro, esta cuestión ha enfrentado a varios autores. Para H. Fränkel ${ }^{22}$ el kolon es una unidad de sentido, mientras que para H. N. Porter ${ }^{23}$, por ejemplo, es sólo una unidad de ritmo. Por su parte G. S. Kirk, en una postura ecléctica ${ }^{24}$, distingue entre "sense-cola» y "rhytmical-cola» ${ }^{25}$, lo que le lleva a considerar una única cesura fundamental en el verso situada en el tercer kolon, trocaica o pentemímeres. Está claro, entonces, que el problema de la definición de la cesura está en intima interrelación con la definición del kolon. Por ello es preciso enfocar los dos conceptos desde la misma óptica.

De nuevo la clave vuelve a estar en la consideración del ritmo y del sentido del verso. Sobre el primer punto no hay, en principio, ningún problema: todo verso se estructura métricamente en una serie de unidades menores, kola, delimitadas por un final de palabra, cesura. Ahora bien, el segundo punto se presta a interpretaciones contradictorias. Nuestro propósito, sin embargo, es dar la importancia que se merece al aspecto sintáctico y de sentido a la hora de poner una cesura en el verso griego, es decir, conjugar el plano del significante con el del significado.

Nos parece muy acertada la relación que Fränkel ${ }^{26}$ establece entre

${ }^{21}$ "The Doctrine of the Caesura, a philological Ghost», AJPh 45, 1924, pp. 329350 .

22 "Der homerische und der kallimachische Hexameter", NGG 1926, pp. 1-33, trabajo rehecho en 1955 y ampliado en su tercera edición de 1968, cf. Wege und Formen fruhgriechischen Denkens, Munich $1968_{3}$, pp. 100-156.

${ }_{23}$ "The early Greek Hexameter», YCS 12, 1951, p. 16.

${ }^{24}$ El defecto de la crítica de Kirk al trabajo de Fränkel lo pone de relieve W. B. Ingalls en uno de sus artículos, "The Structure of the Homeric Hexameter: a Review", Phoenix 24, 1970, pp. 1-12.

${ }^{25}$ G. S. Kirk, "The Structure of the Homeric Hexameter", YCS 20, 1966, pp. 76104, y también The Iliad. A comentary, Cambridge 1985, pp. 18-24.

${ }_{26} \mathrm{Op}$. cit. 
kolon y sintaxis, y la calificación de "microestrofa» aplicada al hexámetro. Esta relación está apoyada sobre la propia génesis del verso. Son numerosas las expresiones formularias que se adecuan a la estructura colométrica del verso, y que ocupan los espacios definidos por las cesuras $^{27}$. Además, según L. E. Rossi ${ }^{28}$, estas expresiones han determinado la estructura del verso hasta el punto de que en el hexámetro el último kolon es el más largo, debido a que es el lugar privilegiado para las cesuras. Además está el hecho del encabalgamiento, que se articula entre las cesuras finales de un verso y las del comienzo de otro ${ }^{29}$ :

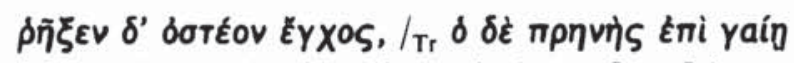

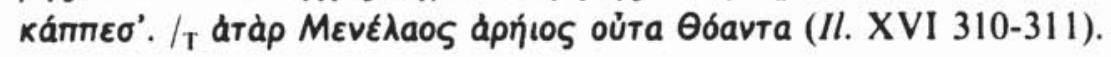

Es éste un caso muy claro donde la cesura se adapta a la realidad sintáctica del verso, pues la frase se organiza entre la cesura trocaica del primer verso y la tritemímeres del segundo.

B. Glavičić ${ }^{30}$, por su parte, en su estudio del tercer kolon en Homero observa que en un gran número de casos la oración comienza a principio de verso y acaba en la diéresis bucólica o en la cesura heptemímeres. Igualmente S. E. Bassett ${ }^{31}$ observa que las cesuras heptemimeres y bucólica introducen diversas cláusulas sintácticas, que sirven para separar la parte esencial de la narración del resto del verso: sintagmas iniciados por una conjunción o relativo, sobre todo kaí, preposiciones, adjetivos o participios en aposición, epítetos, etc...:

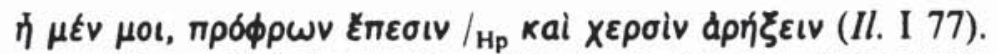

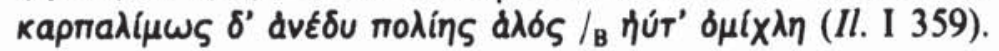

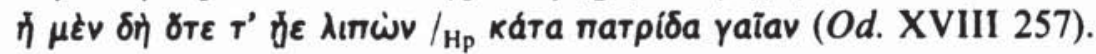

De acuerdo con la definición de Fränkel ${ }^{32}$ las cesuras son «Sinnesfugen», uniones de sentido, que delimitan cada una de las unidades de

${ }^{27}$ J. B. Hainsworth, The Flexibility of the Homeric Formula, Oxford 1968, p. 131 ss.

28 "Estensione e valore del colon nell'esametro omerico". StudUrb 39, 1965, pp. 235-273, p. 270.

${ }^{29}$ Cf. G. S. Kirk, "Verse-Structure and Sentence-Structure in Homer", YCS 20, 1966, pp. 105-152; G. R. McLennan, «Enjambement in the Hymns of Callimachus», Hermes 102, 1974, pp. 200-206; D. L. Clayman y T. van Nortwick, «Enjambement in Greek Hexameter Poetry", TAPhA 107, 1977, pp. 85-92.

${ }_{30}$ "O sintaktičkim osobitostima Homerova trečega kolona», ZAnt 18, 1968, pp. 61-198, y "Homerov treçi kolon kao sintaktiçka cjelina», ZAnt 19, 1969, pp. 175206.

${ }^{31}$ "Notes on the Bucolic Diaeresis», TAPhA 36, 1905, pp. 111-124, y «The Hepthemimeral Caesura", TAPhA 48, 1917, pp. 85-110.

${ }_{32}$ Op. cit. 
sentido. Y lógicamente la unidad mínima de sentido es aquella que consta sólo de una palabra ${ }^{33}$. De ahí la tendencia a establecer el mayor número de cesuras en el hexámetro, tantas como "Word-end" ${ }^{34}$.

Sin embargo, esto sólo es posible en versos que contengan enumeraciones, tal es el caso, por ejemplo, de las sucesiones de nombres propios del conocido catálogo de las naves de Homero $^{35}$ y de la Teogonía de Hesíodo ${ }^{36}$ :

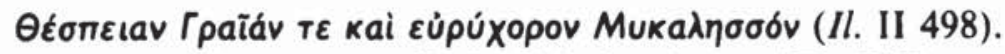

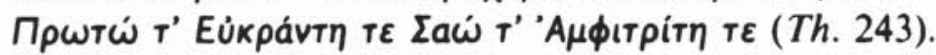

Estos ejemplos suponen un caso extremo de aprovechamiento de las cesuras, ya que lo normal es que el verso se estructure en sintagmas, no en palabras, desde el punto de vista del sentido. $Y$ es aquí donde intervienen razones de tipo subjetivo y de índole estilística a la hora de situar una cesura. Sobre la base de un sistema tipo y modélico de cesuras se organiza el material sintáctico, lo que da lugar a desplazamientos de estas cesuras preestablecidas ${ }^{37}$ para adaptar los contenidos al metro.

No obstante hay cesuras que van acompañadas de puntuación, «starke Sinneschnitte» ${ }^{38}$, lo que las convierte en elemento fundamental para el sentido del verso. Según vemos en la tabla presentada por Beck, los lugares más frecuentes de la puntuación son precisamente la cesura trocaica, la pentemímeres, la diéresis bucólica y la tritemímeres, en este orden ${ }^{39}$. No es una mera casualidad, sino que es una de las pruebas de la relación entre cesura y sentido del verso. En estos puntos coincide plenamente el ritmo y el sentido; aquí las cesuras muestran su función en su doble valor.

Ahora bien, la realización concreta de cada uno de los versos no es siempre así. A veces es dificil asignar a la cesura un papel importante en el sentido del verso, ya que, como hemos indicado, intervienen razo-

${ }^{33}$ Glavičić, "O sintaktičkim...", observa que más del $50 \%$ de los versos de Homero presentan el tercer kolon compuesto por una sola palabra, con una unidad sintáctica suficientemente autónoma.

${ }^{34}$ En el hexámetro de los Oráculos Sibilinos se puede ver claramente esta tendencia a hacer coincidir el fin de palabra con los lugares tradicionales de cesura, de modo que el verso parece construido palabra a palabra, una por cada segmento acotado por cesuras: cf. nuestro trabajo El hexámetro de los Oráculos Sibilinos, Amsterdam 1992, p. 134 ss.

${ }^{35}$ Il. II 484-760, en especial los versos 497 ss.

36 Th. $241-247$ y 355 .

${ }^{37}$ Lo que Fränkel llama cesuras desplazadas, cf. op. cit.

${ }^{38}$ Beck, op. cit., p. 12.

39 Vid. datos estadísticos para Homero en Beck, op. cit., p. 12. 
nes subjetivas y estilísticas ${ }^{40}$. De todas formas no se puede dejar a un lado el contenido, y así, aunque la estructura rítmica del verso pida una cesura, sin embargo no podremos ir en contra de la sintaxis ${ }^{41}$, y por tanto no habrá lugar para una determinada cesura. Detengámonos unos instantes en esta cuestión, en el problema de fin de palabra en los lugares habituales de cesura.

Muchos versos se articulan sintácticamente a través de la cesura media y la diéresis bucólica, y, además, presentan la posibilidad de corte en la cesura heptemímeres para destacar un nombre de un adjetivo, un complemento nominal de un sustantivo, el sujeto u objeto de su verbo correspondiente, etc...:

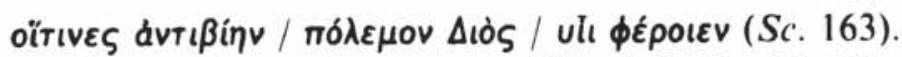

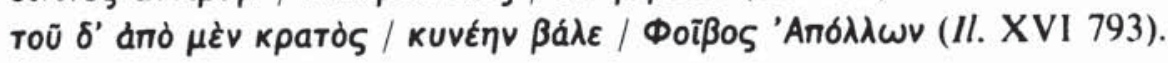

Serían, en principio, razones estilísticas y subjetivas las que nos llevarian a interpretar un corte tras kuvénv como un simple fin de palabra o como cesura propiamente dicha.

Asimismo, en el primer segmento del verso la función de las cesuras es separar o destacar algún elemento del resto de la exposición: el verbo, el primer término de una enumeración, imperativos y vocativos, el sustantivo de su adjetivo, etc...:

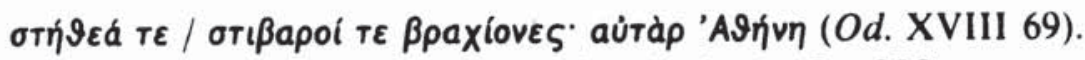

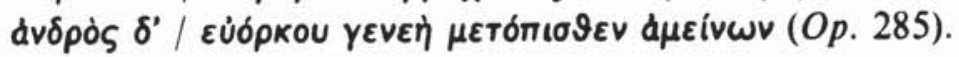

También son muy numerosas las conjunciones y expresiones adverbiales que ocupan estas posiciones, si bien el sentido hace imposible el corte:

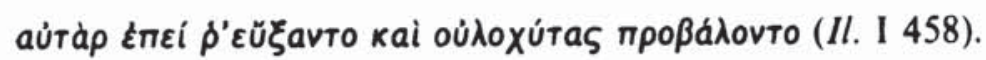

Teóricamente podría haber cesura tritemímeres tras $\varepsilon \pi \varepsilon \dot{i}$, sin embargo la sintaxis lo impide.

Podríamos seguir poniendo ejemplos de esta relación entre metro y sentido o, mejor dicho, entre la cesura y el contenido del verso, aunque

${ }^{40}$ Un ejemplo de este empleo estilístico y retórico de la cesura puede verse en el artículo de F. L. Clark, "Caesural emphasis in the Iliad", CJ 9, 1913-14, pp. 61-66.

${ }_{41}$ No entramos aquí en el problema de las apositivas y su relación con las cesuras, para lo que nos remitimos al trabajo de A. M. Devine y L. D. Stephens, "The Greek appositives: toward a linguistically adequate definition of caesura and bridge», CPh 73, 1978, pp. 314-328. 
consideramos que éstos son suficientes para nuestro propósito. Está claro que la función de las cesuras es ajustar el sentido de la frase a la estructura métrica del verso. El contenido del verso se organiza de acuerdo con las cesuras. Esto es un hecho que está ahí, latente en cada momento, es algo que nos aparece siempre que analizamos un verso desde el punto de vista métrico, siempre que intentamos colocar una cesura.

A la vista de estos ejemplos, parece necesario distinguir entre aquellas cesuras fundamentales para el sentido, y aquellas que son meramente estilísticas o retóricas. Evidentemente hay una serie de pausas que podemos considerar principales, por su estrecha relación con el contenido (estén o no apoyados por puntuación), mientras que otras son accesorias y tienen un fin estilístico además de meramente métrico o estructural. Esto lo podemos comprobar en el siguiente ejemplo:

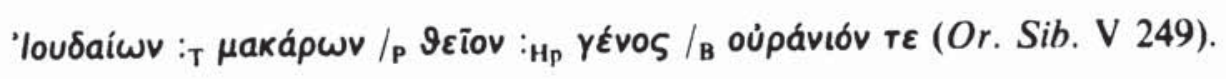

Las cesuras fundamentales (notadas con /) son la pentemímeres y la bucólica, que dividen el verso en tres miembros sintácticos. Junto a ellas pueden admitirse, con un valor secundario, otros dos cortes más, tritemímeres y heptemímeres (las señaladas con :), para destacar desde el punto de vista estilístico determinados elementos de cada uno de los sintagmas.

No obstante en muchas ocasiones nos vamos a encontrar con contradicciones, tanto en la sintaxis como en la métrica, ya que con frecuencia las unidades de sentido no coinciden totalmente con las unidades rítmicas ${ }^{42}$, si bien las estadísticas muestran un alto grado de coincidencia entre kolon métrico y kolon sintáctico o, al menos, los kola de sentido generalmente acaban en la misma posición que los sintácticos ${ }^{43}$.

Llegados a este punto, creemos que es momento de recapitular y sacar conclusiones de las reflexiones que hemos hecho a lo largo de este artículo:

1. Debemos distinguir entre "word-end", simple fin de palabra, y cesura, es decir, fin de palabra con implicaciones en la estructura, el ritmo, la fonética, la prosodia y la sintaxis del verso.

2. La cesura está en estrecha relación con el contenido y sentido del verso, o al menos no está en contradicción con él. Diversos hechos,

\footnotetext{
${ }^{42}$ Beck, op. cit., p. 7 ss. recoge varios ejemplos donde la colometría del verso viola la estructura sintáctica y lógica del sentido de la frase.

${ }^{43}$ Cf. L. Cañigral, Estudios estilistico-métricos sobre Apolonio de Rodas, Madrid 1988, en especial pp. 88-117.
} 
tales como el encabalgamiento, la puntuación, las cláusulas y expresiones formularias, etc... evidencian esta cohesión entre metro y sintaxis.

3. No todas las cesuras tienen la misma importancia o intensidad, sino que la realidad concreta de cada verso asigna a unas cesuras un papel fundamental en su estructura y contenido, mientras que otras no son más que unos cortes secundarios o complementarios.

4. Ahora bien, esta relación entre metro y sintaxis no implica una pausa en la recitación, sino una interrelación con el sentido, dado que nos es prácticamente imposible recomponer la realización concreta del verso griego.

5. Tampoco podemos ignorar las razones estilísticas y retóricas que condicionan la ubicación de las cesuras en el verso, ya que una cosa es el esquema ideal de las cesuras de un verso, y otra es su realización concreta. Este punto es reconocido incluso desde un punto de vista estructural por S. Mariner, para quien la cesura, a pesar de ser un hecho sistemático y mecánico, adquiere en manos del poeta una amplia función estilística ${ }^{44}$.

En resumen, la cesura es un fenómeno métrico-sintáctico con implicación en el plano del ritmo y del significado del verso. Es, por tanto, una prueba más de esa relación tan extendida en la poesía griega entre forma y contenido. Podemos concluir con palabras de J. S. Lasso de la Vega, para el caso concreto del hexámetro, diciendo que «el ordenamiento interno del contenido ejerció influencia tanta sobre la conformación externa del hexámetro, que nosotros hoy podemos, sobre la letra muerta del texto, descubrir esas «reglas» de la recitación en la que verso y palabra se consustantivan ${ }^{45}$.

Jesús María Nieto Ibáñez

44 «Lo que podía tenerse por algo mecánico, más o menos impuesto por el verso mismo, se revela como cosa potestativa, administrable por el poeta según su auténtico gusto personal y, por consiguiente, computable a efectos estilísticos en todo su valor: entra de lleno en el gran campo de las relaciones entre elementos métricos y significativos", cf. Mariner, op. cit., p. 324.

${ }_{45}$ J. S. Lasso de la Vega, «Perspectivas actuales en el campo de la métrica griega», EC 84, 1979, p. 226. 